\title{
A Note on Entire Functions That Share Two Small Functions
}

\author{
Jun-Fan Chen \\ Department of Mathematics, Fujian Normal University, Fuzhou, Fujian 350007, China
}

Correspondence should be addressed to Jun-Fan Chen; junfanchen@163.com

Received 10 April 2014; Accepted 16 September 2014; Published 19 October 2014

Academic Editor: V. Ravichandran

Copyright (C) 2014 Jun-Fan Chen. This is an open access article distributed under the Creative Commons Attribution License, which permits unrestricted use, distribution, and reproduction in any medium, provided the original work is properly cited.

This note is to show that if $f$ is a nonconstant entire function that shares two pairs of small functions ignoring multiplicities with its first derivative $f^{\prime}$, then there exists a close linear relationship between $f$ and $f^{\prime}$. This result is a generalization of some results obtained by Rubel and Yang, Mues and Steinmetz, Zheng and Wang, and Qiu. Moreover, examples are provided to show that the conditions in the result are sharp.

\section{Introduction and Main Result}

Throughout this paper, we use standard notations in the Nevanlinna theory (see, e.g., [1-4]). Let $f(z)$ be a meromorphic function. Here and in the following the word "meromorphic" means meromorphic in the whole complex plane. We denote by $S(r, f)$ any real function of growth $o(T(r, f))$ as $r \rightarrow \infty$ outside of a possible exceptional set of finite linear measure. The meromorphic function $a$ is called a small function with respect to $f$ provided that $T(r, a)=S(r, f)$.

Let $f$ and $g$ be two nonconstant meromorphic functions, and let $a$ and $b$ be two small functions with respect to $f$ and $g$. If the zeros of $f-a$ and $g-b$ coincide in locations and multiplicities, then we say that $f$ and $g$ share the pair of small functions $(a, b)$ CM (counting multiplicities); if we do not consider the multiplicities, then $f$ and $g$ are said to share the pair of small functions $(a, b)$ IM (ignoring multiplicities). We see that $f$ and $g$ share the pair of small functions $(a, a) \mathrm{CM}$ if and only if $f$ and $g$ share the small function $a \mathrm{CM}$, and $f$ and $g$ share the pair of small functions $(a, a)$ IM if and only if $f$ and $g$ share the small function $a$ IM. The same argument applies in the case when $a$ and $b$ are two values in the extended plane.

Moreover, we introduce the following notations. Denote the set of those points $z \in \mathbf{C}$ by $S_{(m, n)}\left(a_{1}, a_{2}\right)$ such that $z$ is a zero of $f-a_{1}$ of multiplicity $m$ and a zero of $f^{\prime}-a_{2}$ of multiplicity $n$. The set $S_{(m, n)}\left(b_{1}, b_{2}\right)$ can be similarly defined. Now the notations $N_{(m, n)}\left(r, 1 /\left(f-a_{1}\right)\right)$ and $\bar{N}_{(m, n)}(r, 1 /(f-$ $\left.a_{1}\right)$ ) denote the counting function and the reduced counting function of $f$ with respect to the set $S_{(m, n)}\left(a_{1}, a_{2}\right)$, respectively. The notations $N_{(m, n)}\left(r, 1 /\left(f^{\prime}-a_{2}\right)\right)$ and $\bar{N}_{(m, n)}\left(r, 1 /\left(f^{\prime}-a_{2}\right)\right)$ can be similarly defined.

Many mathematicians have been interested in the value distribution of different expressions of an entire or meromorphic function and obtained a lot of fruitful and significant results. When dealing with an entire function $f$ and its derivative $f^{\prime}$, Rubel and Yang [5] proved the following.

Theorem A. Let $f$ be a nonconstant entire function, and let a and $b$ be distinct finite complex numbers. If $f$ and $f^{\prime}$ share $a$ and $b C M$, then $f \equiv f^{\prime}$.

Mues and Steinmetz [6] improved Theorem A and obtained the following.

Theorem B. Let $f$ be a nonconstant entire function, and let a and $b$ be distinct finite complex numbers. If $f$ and $f^{\prime}$ share $a$ and $b I M$, then $f \equiv f^{\prime}$.

When the values $a$ and $b$ were replaced by two small functions related to $f$, Zheng and Wang [7] proved the following.

Theorem C. Let $f$ be a nonconstant entire function, and let a and $b$ be distinct small functions with respect to $f$. If $f$ and $f^{\prime}$ share $a$ and $b C M$, then $f \equiv f^{\prime}$.

Recently, Qiu [8] proved the following result which was an improvement of Theorem C. 
Theorem D. Let $f$ be a nonconstant entire function, and let a and $b$ be distinct small functions with respect to $f$. If $f$ and $f^{\prime}$ share $a$ and $b I M$, then $f \equiv f^{\prime}$.

This paper is concerned with what can be said when the IM shared small function is replaced by the IM shared the pair of small functions in Theorem D. In fact, we prove the following result by using the method of [8], which generalizes the above theorems from the point of view of shared pairs.

Theorem 1. Let $f$ be a nonconstant entire function, and let $a_{1}$, $a_{2}, b_{1}$, and $b_{2}$ be four small functions of $f$ such that none of them is identically equal to $\infty$ and $a_{1} \neq \equiv b_{1}, a_{2} \neq \equiv b_{2}$. If $f$ and $f^{\prime}$ share $\left(a_{1}, a_{2}\right)$ and $\left(b_{1}, b_{2}\right)$ IM, then $\left(a_{2}-b_{2}\right) f-\left(a_{1}-b_{1}\right) f^{\prime}+$ $a_{1} b_{2}-a_{2} b_{1} \equiv 0$.

Remark 2. Let $a_{1} \equiv a_{2}$ and $b_{1} \equiv b_{2}$. Then by Theorem 1 we can get Theorem $\mathrm{D}$.

Remark 3. Theorem 1 shows that a nonconstant entire function sharing two pairs of small functions ignoring multiplicities with its first derivative implies that there exists a close linear relationship between them.

Example 4 (see [9]). Let $f=\beta+(\beta-\alpha) /(h-1)$, where

$$
\alpha=-\frac{1}{3} e^{-2 z}-\frac{1}{2} e^{-z}, \quad \beta=-\frac{1}{3} e^{-2 z}+\frac{1}{2} e^{-z}, \quad h=e^{-e^{z}} .
$$

Set $a=\beta^{\prime}, b=\alpha^{\prime}$. Then $T(r, a)=S(r, f)$ and $T(r, b)=$ $S(r, f)$. It is easy to verify that

$$
\begin{aligned}
& f^{\prime}-a=e^{2 z}(f-a)(f-\beta), \\
& f^{\prime}-b=e^{2 z}(f-b)(f-\alpha) .
\end{aligned}
$$

Thus $f$ and $f^{\prime}$ share $(a, a)$ and $(b, b)$ IM, but $f \not \equiv f^{\prime}$. This shows that the conclusion in Theorem 1 is not valid generally for a meromorphic function $f$.

Example 5. Let $f=e^{2 z}+z, a_{1}=2 z, a_{2}=2 z+1, b_{1}=z$, and $b_{2}=z+1$. Then $f$ and $f^{\prime}$ share $\left(a_{1}, a_{2}\right)$ IM but do not share $\left(b_{1}, b_{2}\right)$ IM. Clearly, $\left(a_{2}-b_{2}\right) f-\left(a_{1}-b_{1}\right) f^{\prime}+a_{1} b_{2}-a_{2} b_{1} \not \equiv 0$. This shows that the condition in Theorem 1 that $f$ and $f^{\prime}$ share $\left(a_{1}, a_{2}\right)$ and $\left(b_{1}, b_{2}\right)$ IM cannot be weakened.

\section{Some Lemmas}

Lemma 1. Let $f$ be a nonconstant entire function, and let $a_{1}$, $a_{2}, b_{1}$, and $b_{2}$ be four small functions of $f$ such that none of them is identically equal to $\infty$ and $a_{1} \not \equiv b_{1}, a_{2} \not \equiv b_{2}$. If $f$ and $f^{\prime}$ share $\left(a_{1}, a_{2}\right)$ and $\left(b_{1}, b_{2}\right)$ IM, then $S(r, f)=S\left(r, f^{\prime}\right):=S(r)$.

Proof. Note that $f$ and $f^{\prime}$ share $\left(a_{1}, a_{2}\right)$ and $\left(b_{1}, b_{2}\right)$ IM. By the second fundamental theorem, we get

$$
\begin{aligned}
T(r, f) & \leq \bar{N}\left(r, \frac{1}{f-a_{1}}\right)+\bar{N}\left(r, \frac{1}{f-b_{1}}\right)+S(r, f) \\
& =\bar{N}\left(r, \frac{1}{f^{\prime}-a_{2}}\right)+\bar{N}\left(r, \frac{1}{f^{\prime}-b_{2}}\right)+S(r, f)
\end{aligned}
$$

$$
\begin{aligned}
& \leq 2 T\left(r, f^{\prime}\right)+S(r, f), \\
T\left(r, f^{\prime}\right) & \leq \bar{N}\left(r, \frac{1}{f^{\prime}-a_{2}}\right)+\bar{N}\left(r, \frac{1}{f^{\prime}-b_{2}}\right)+S\left(r, f^{\prime}\right) \\
& =\bar{N}\left(r, \frac{1}{f-a_{1}}\right)+\bar{N}\left(r, \frac{1}{f-b_{1}}\right)+S\left(r, f^{\prime}\right) \\
& \leq 2 T(r, f)+S(r, f)+S\left(r, f^{\prime}\right),
\end{aligned}
$$

which implies from the definition of $S(r, f)$ that $S(r, f)=$ $S\left(r, f^{\prime}\right)$ and $S\left(r, f^{\prime}\right)=S(r, f)$, respectively.

This completes the proof of Lemma 1.

Lemma 2. Let $f$ be a nonconstant entire function, and let $a_{1}$, $a_{2}, b_{1}$, and $b_{2}$ be four small functions of $f$ such that none of them is identically equal to $\infty$ and $a_{1} \neq \equiv b_{1}, a_{2} \neq \equiv b_{2}$. If $f$ and $f^{\prime}$ share $\left(a_{1}, a_{2}\right)$ and $\left(b_{1}, b_{2}\right)$ IM, then

$$
m(r, f)=\bar{N}\left(r, \frac{1}{f-a_{1}}\right)+\bar{N}\left(r, \frac{1}{f-b_{1}}\right)+S(r)
$$

provided that $\left(a_{2}-b_{2}\right) f-\left(a_{1}-b_{1}\right) f^{\prime}+a_{1} b_{2}-a_{2} b_{1} \neq \equiv 0$.

Proof. Note that

$$
\begin{aligned}
& \left(a_{2}-b_{2}\right) f-\left(a_{1}-b_{1}\right) f^{\prime}+a_{1} b_{2}-a_{2} b_{1} \neq 0, \\
& \left(a_{2}-b_{2}\right) f-\left(a_{1}-b_{1}\right) f^{\prime}+a_{1} b_{2}-a_{2} b_{1} \\
& \equiv\left(a_{2}-b_{2}\right)\left(f-a_{1}\right)-\left(a_{1}-b_{1}\right)\left(f^{\prime}-a_{2}\right), \\
& \left(a_{2}-b_{2}\right) f-\left(a_{1}-b_{1}\right) f^{\prime}+a_{1} b_{2}-a_{2} b_{1} \\
& \equiv\left(a_{2}-b_{2}\right)\left(f-b_{1}\right)-\left(a_{1}-b_{1}\right)\left(f^{\prime}-b_{2}\right) .
\end{aligned}
$$

Since $f$ and $f^{\prime}$ share $\left(a_{1}, a_{2}\right)$ and $\left(b_{1}, b_{2}\right)$ IM, from Lemma 1 , (5)-(7), and the condition that $f$ is entire, we have

$$
\begin{aligned}
& \bar{N}\left(r, \frac{1}{f-a_{1}}\right)+\bar{N}\left(r, \frac{1}{f-b_{1}}\right) \\
& \leq N\left(r, \frac{1}{\left(a_{2}-b_{2}\right) f-\left(a_{1}-b_{1}\right) f^{\prime}+a_{1} b_{2}-a_{2} b_{1}}\right) \\
& \leq T\left(r,\left(a_{2}-b_{2}\right) f-\left(a_{1}-b_{1}\right) f^{\prime}\right)+S(r) \\
&=m\left(r,\left(a_{2}-b_{2}\right) f-\left(a_{1}-b_{1}\right) f^{\prime}\right) \\
& \quad+N\left(r,\left(a_{2}-b_{2}\right) f-\left(a_{1}-b_{1}\right) f^{\prime}\right)+S(r) \\
&=m\left(r,\left(a_{2}-b_{2}\right) f-\left(a_{1}-b_{1}\right) f^{\prime}\right)+S(r) \\
& \leq m\left(r, \frac{\left(a_{2}-b_{2}\right) f-\left(a_{1}-b_{1}\right) f^{\prime}}{f}\right)+m(r, f)+S(r) \\
& \leq m(r, f)+S(r) .
\end{aligned}
$$


On the other hand, by the second fundamental theorem, Lemma 1 , and the condition that $f$ is entire, we get

$$
\begin{aligned}
m(r, f)=T(r, f) \leq & \bar{N}\left(r, \frac{1}{f-a_{1}}\right) \\
& +\bar{N}\left(r, \frac{1}{f-b_{1}}\right)+S(r) .
\end{aligned}
$$

Now (8) and (9) imply

$$
m(r, f)=\bar{N}\left(r, \frac{1}{f-a_{1}}\right)+\bar{N}\left(r, \frac{1}{f-b_{1}}\right)+S(r) .
$$

This completes the proof of Lemma 2.

Lemma 3. Let $f$ be a nonconstant entire function, and let $a_{1}$, $a_{2}, b_{1}$, and $b_{2}$ be four small functions of $f$ such that none of them is identically equal to $\infty$ and $a_{1} \neq \equiv b_{1}, a_{2} \neq \equiv b_{2}$. Suppose that $f$ and $f^{\prime}$ share $\left(a_{1}, a_{2}\right)$ and $\left(b_{1}, b_{2}\right)$ IM. Set

$$
\begin{array}{r}
\alpha=\left(a_{1}^{\prime}-b_{1}^{\prime}\right)\left(f-a_{1}\right)-\left(a_{1}-b_{1}\right)\left(f^{\prime}-a_{1}^{\prime}\right) \\
=\left(a_{1}^{\prime}-b_{1}^{\prime}\right)\left(f-b_{1}\right)-\left(a_{1}-b_{1}\right)\left(f^{\prime}-b_{1}^{\prime}\right), \\
\beta=\left(a_{2}^{\prime}-b_{2}^{\prime}\right)\left(f^{\prime}-a_{2}\right)-\left(a_{2}-b_{2}\right)\left(f^{\prime \prime}-a_{2}^{\prime}\right) \\
=\left(a_{2}^{\prime}-b_{2}^{\prime}\right)\left(f^{\prime}-b_{2}\right)-\left(a_{2}-b_{2}\right)\left(f^{\prime \prime}-b_{2}^{\prime}\right), \\
g=\frac{\alpha\left[\left(a_{2}-b_{2}\right) f-\left(a_{1}-b_{1}\right) f^{\prime}+a_{1} b_{2}-a_{2} b_{1}\right]}{\left(f-a_{1}\right)\left(f-b_{1}\right)}, \\
h=\frac{\beta\left[\left(a_{2}-b_{2}\right) f-\left(a_{1}-b_{1}\right) f^{\prime}+a_{1} b_{2}-a_{2} b_{1}\right]}{\left(f^{\prime}-a_{2}\right)\left(f^{\prime}-b_{2}\right)}, \\
\gamma_{i}=a_{2}+i\left(a_{2}-b_{2}\right), \quad(i=1,2) .
\end{array}
$$

If $\left(a_{2}-b_{2}\right) f-\left(a_{1}-b_{1}\right) f^{\prime}+a_{1} b_{2}-a_{2} b_{1} \neq \equiv 0$, then

(i) $T(r, g)=S(r)$,

(ii) $T(r, h) \leq T(r, f)-N\left(r, 1 /\left(f^{\prime}-\gamma_{i}\right)\right)+S(r)$ for $i=1,2$.

Proof. Since $f$ and $f^{\prime}$ share $\left(a_{1}, a_{2}\right)$ and $\left(b_{1}, b_{2}\right)$ IM, by Lemma 1 we know $S(r, f)=S\left(r, f^{\prime}\right):=S(r)$. Noting

$$
\begin{aligned}
& \frac{\alpha}{f-a_{1}}=\frac{\left(a_{1}^{\prime}-b_{1}^{\prime}\right)\left(f-a_{1}\right)-\left(a_{1}-b_{1}\right)\left(f^{\prime}-a_{1}^{\prime}\right)}{f-a_{1}} \\
&=a_{1}^{\prime}-b_{1}^{\prime}-\left(a_{1}-b_{1}\right) \frac{f^{\prime}-a_{1}^{\prime}}{f-a_{1}}, \\
& \frac{\alpha}{f-b_{1}}=\frac{\left(a_{1}^{\prime}-b_{1}^{\prime}\right)\left(f-b_{1}\right)-\left(a_{1}-b_{1}\right)\left(f^{\prime}-b_{1}^{\prime}\right)}{f-b_{1}} \\
&=a_{1}^{\prime}-b_{1}^{\prime}-\left(a_{1}-b_{1}\right) \frac{f^{\prime}-b_{1}^{\prime}}{f-b_{1}}, \\
& \frac{1}{\left(f-a_{1}\right)\left(f-b_{1}\right)}=\frac{1}{a_{1}-b_{1}}\left(\frac{1}{f-a_{1}}-\frac{1}{f-b_{1}}\right),
\end{aligned}
$$

$$
\begin{aligned}
& \frac{\beta}{f^{\prime}-a_{2}}=\frac{\left(a_{2}^{\prime}-b_{2}^{\prime}\right)\left(f^{\prime}-a_{2}\right)-\left(a_{2}-b_{2}\right)\left(f^{\prime \prime}-a_{2}^{\prime}\right)}{f^{\prime}-a_{2}} \\
&=a_{2}^{\prime}-b_{2}^{\prime}-\left(a_{2}-b_{2}\right) \frac{f^{\prime \prime}-a_{2}^{\prime}}{f^{\prime}-a_{2}}, \\
& \frac{\beta}{f^{\prime}-b_{2}}=\frac{\left(a_{2}^{\prime}-b_{2}^{\prime}\right)\left(f^{\prime}-b_{2}\right)-\left(a_{2}-b_{2}\right)\left(f^{\prime \prime}-b_{2}^{\prime}\right)}{f^{\prime}-b_{2}} \\
&=a_{2}^{\prime}-b_{2}^{\prime}-\left(a_{2}-b_{2}\right) \frac{f^{\prime \prime}-b_{2}^{\prime}}{f^{\prime}-b_{2}}, \\
& \frac{1}{\left(f^{\prime}-a_{2}\right)\left(f^{\prime}-b_{2}\right)}=\frac{1}{a_{2}-b_{2}}\left(\frac{1}{f^{\prime}-a_{2}}-\frac{1}{f^{\prime}-b_{2}}\right),
\end{aligned}
$$

and the lemma of the logarithmic derivative, we obtain

$$
\begin{gathered}
m\left(r, \frac{\alpha}{f-a_{1}}\right)=S(r), \quad m\left(r, \frac{\alpha}{f-b_{1}}\right)=S(r), \\
m\left(r, \frac{\alpha}{\left(f-a_{1}\right)\left(f-b_{1}\right)}\right)=S(r), \\
m\left(r, \frac{\beta}{f^{\prime}-a_{2}}\right)=S(r), \quad m\left(r, \frac{\beta}{f^{\prime}-b_{2}}\right)=S(r), \\
m\left(r, \frac{\beta}{\left(f^{\prime}-a_{2}\right)\left(f^{\prime}-b_{2}\right)}\right)=S(r) .
\end{gathered}
$$

Clearly, $\alpha \neq \equiv 0$ and $\beta \not \equiv 0$. Otherwise from (11) and (12) we have $f=a_{1}+C_{1}\left(a_{1}-b_{1}\right)$ and $f^{\prime}=a_{2}+C_{2}\left(a_{2}-b_{2}\right)$ for nonzero constants $C_{1}, C_{2}$, which implies that $T(r, f)=S(r)$ and $T\left(r, f^{\prime}\right)=S(r)$, a contradiction. Then by using a similar method we can deduce that $g \not \equiv 0$ and $h \neq \equiv 0$. It is easy to see by (11) if any zero of $f-a_{1}\left(f-b_{1}\right)$ of multiplicity $l$ is not the pole of $a_{1}-b_{1}$ and is not the zero of $a_{1}-b_{1}$, then it must be a zero of $\alpha$ of multiplicity $l-1$ at least. Thus from (6), (7), (11), (13), the condition that $f$ and $f^{\prime}$ share $\left(a_{1}, a_{2}\right)$ and $\left(b_{1}, b_{2}\right) \mathrm{IM}$, and the condition that $f$ is entire, we get

$$
N(r, g)=S(r) .
$$

Likewise,

$$
N(r, h)=S(r) .
$$

Now by (13) and (17) together with

$$
\frac{\alpha f}{\left(f-a_{1}\right)\left(f-b_{1}\right)}=\frac{\alpha}{f-b_{1}}+\frac{a_{1} \alpha}{\left(f-a_{1}\right)\left(f-b_{1}\right)},
$$

it follows that

$$
\begin{aligned}
m(r, g) \leq & m\left(r, \frac{\alpha\left[\left(a_{2}-b_{2}\right) f-\left(a_{1}-b_{1}\right) f^{\prime}\right]}{\left(f-a_{1}\right)\left(f-b_{1}\right)}\right) \\
& +m\left(r, \frac{\alpha\left(a_{1} b_{2}-a_{2} b_{1}\right)}{\left(f-a_{1}\right)\left(f-b_{1}\right)}\right)+\log 2
\end{aligned}
$$




$$
\begin{aligned}
\leq & m\left(r, \frac{\alpha f}{\left(f-a_{1}\right)\left(f-b_{1}\right)}\right) \\
& +m\left(r, \frac{\left(a_{2}-b_{2}\right) f-\left(a_{1}-b_{1}\right) f^{\prime}}{f}\right)+S(r)
\end{aligned}
$$$$
\leq S(r) \text {. }
$$

Thus from this and (19) we have

$$
T(r, g)=S(r)
$$

implying (i). Next, it is easy to see that $\gamma_{i} \not \equiv a_{2}$ and $\gamma_{i} \not \equiv$ $b_{2}(i=1,2)$. For $i=1,2$, by (14), (18), $a_{1} \neq \equiv b_{1}, a_{2} \neq \equiv b_{2}$, and the condition that $f$ is entire, we have

$$
\begin{aligned}
& m(r, h) \leq m\left(r, \frac{\beta\left[\left(a_{2}-b_{2}\right) f-\left(a_{1}-b_{1}\right) f^{\prime}\right]}{\left(f^{\prime}-a_{2}\right)\left(f^{\prime}-b_{2}\right)}\right) \\
& +m\left(r, \frac{\beta\left(a_{1} b_{2}-a_{2} b_{1}\right)}{\left(f^{\prime}-a_{2}\right)\left(f^{\prime}-b_{2}\right)}\right)+\log 2 \\
& \leq m\left(r, \frac{\beta\left(f^{\prime}-\gamma_{i}\right)}{\left(f^{\prime}-a_{2}\right)\left(f^{\prime}-b_{2}\right)}\right. \\
& \left.\times \frac{\left(a_{2}-b_{2}\right) f-\left(a_{1}-b_{1}\right) f^{\prime}}{f^{\prime}-\gamma_{i}}\right)+S(r) \\
& \leq m\left(r, \frac{\beta}{f^{\prime}-b_{2}}\right)+m\left(r, \frac{\beta\left(a_{2}-\gamma_{i}\right)}{\left(f^{\prime}-a_{2}\right)\left(f^{\prime}-b_{2}\right)}\right) \\
& +m\left(r, \frac{\left(a_{2}-b_{2}\right) f-\left(a_{1}-b_{1}\right) f^{\prime}}{f^{\prime}-\gamma_{i}}\right)+S(r) \\
& \leq m\left(r, \frac{\left(\left(a_{2}-b_{2}\right) /\left(a_{1}-b_{1}\right)\right) f-f^{\prime}}{f^{\prime}-\gamma_{i}}\right)+S(r) \\
& \leq m\left(r, \frac{\left(\left(a_{2}-b_{2}\right) /\left(a_{1}-b_{1}\right)\right) f-\gamma_{i}^{\prime}}{f^{\prime}-\gamma_{i}}-1\right)+S(r) \\
& \leq m\left(r, \frac{f^{\prime}-\gamma_{i}}{\left(\left(a_{2}-b_{2}\right) /\left(a_{1}-b_{1}\right)\right) f-\gamma_{i}}\right) \\
& +N\left(r, \frac{f^{\prime}-\gamma_{i}}{\left(\left(a_{2}-b_{2}\right) /\left(a_{1}-b_{1}\right)\right) f-\gamma_{i}}\right) \\
& -N\left(r, \frac{\left(\left(a_{2}-b_{2}\right) /\left(a_{1}-b_{1}\right)\right) f-\gamma_{i}}{f^{\prime}-\gamma_{i}}\right)+S(r) \\
& \leq m\left(r, \frac{1}{\left(a_{2}-b_{2}\right) /\left(a_{1}-b_{1}\right)}\right. \\
& \left.\times \frac{f^{\prime}-\gamma_{i}}{f-\left(\left(a_{1}-b_{1}\right) /\left(a_{2}-b_{2}\right)\right) \gamma_{i}}\right) \\
& +N\left(r, f^{\prime}-\gamma_{i}\right)
\end{aligned}
$$

$$
\begin{aligned}
& +N\left(r, \frac{1}{\left(\left(a_{2}-b_{2}\right) /\left(a_{1}-b_{1}\right)\right) f-\gamma_{i}}\right) \\
& -N\left(r, \frac{a_{2}-b_{2}}{a_{1}-b_{1}} f-\gamma_{i}\right)-N\left(r, \frac{1}{f^{\prime}-\gamma_{i}}\right)+S(r) \\
& \leq m\left(r, \frac{f^{\prime}-\left(\left(\left(a_{1}-b_{1}\right) /\left(a_{2}-b_{2}\right)\right) \gamma_{i}\right)^{\prime}}{f-\left(\left(a_{1}-b_{1}\right) /\left(a_{2}-b_{2}\right)\right) \gamma_{i}}\right)
\end{aligned}
$$$$
+m\left(r, \frac{\left(\left(\left(a_{1}-b_{1}\right) /\left(a_{2}-b_{2}\right)\right) \gamma_{i}\right)^{\prime}-\gamma_{i}}{f-\left(\left(a_{1}-b_{1}\right) /\left(a_{2}-b_{2}\right)\right) \gamma_{i}}\right)
$$$$
+N\left(r, \frac{1}{\left(\left(a_{2}-b_{2}\right) /\left(a_{1}-b_{1}\right)\right) f-\gamma_{i}}\right)
$$$$
-N\left(r, \frac{1}{f^{\prime}-\gamma_{i}}\right)+S(r)
$$$$
\leq m\left(r, \frac{1}{f-\left(\left(a_{1}-b_{1}\right) /\left(a_{2}-b_{2}\right)\right) \gamma_{i}}\right)
$$$$
+N\left(r, \frac{1}{\left(\left(a_{2}-b_{2}\right) /\left(a_{1}-b_{1}\right)\right) f-\gamma_{i}}\right)
$$$$
-N\left(r, \frac{1}{f^{\prime}-\gamma_{i}}\right)+S(r)
$$$$
\leq m\left(r, \frac{1}{\left(\left(a_{2}-b_{2}\right) /\left(a_{1}-b_{1}\right)\right) f-\gamma_{i}}\right)
$$$$
+N\left(r, \frac{1}{\left(\left(a_{2}-b_{2}\right) /\left(a_{1}-b_{1}\right)\right) f-\gamma_{i}}\right)
$$$$
-N\left(r, \frac{1}{f^{\prime}-\gamma_{i}}\right)+S(r)
$$$$
\leq T(r, f)-N\left(r, \frac{1}{f^{\prime}-\gamma_{i}}\right)+S(r) \text {. }
$$

Thus from (20) and (24) it follows that

$$
T(r, h) \leq T(r, f)-N\left(r, \frac{1}{f^{\prime}-\gamma_{i}}\right)+S(r), \quad i=1,2 .
$$

This proves (ii) and completes the proof of Lemma 3.

Lemma 4 (see [10]; cf. $[11,12]$ ). Let $f$ be a nonconstant meromorphic function, and let $f^{n} P(f)=Q(f)$, where $P(f)$ and $Q(f)$ are differential polynomials in $f$ and the degree of $Q(f)$ is at most $n$. Then

$$
m(r, P(f))=S(r, f) .
$$


Lemma 5. Let $f$ be a nonconstant entire function, and let $a_{1}$, $a_{2}, b_{1}$, and $b_{2}$ be four small functions of $f$ such that none of them is identically equal to $\infty$ and $a_{1} \not \equiv b_{1}, a_{2} \neq \equiv b_{2}$. Suppose that $f$ and $f^{\prime}$ share $\left(a_{1}, a_{2}\right)$ and $\left(b_{1}, b_{2}\right)$ IM. If

$$
m(r, f)=m\left(r, f^{\prime}\right)+S(r, f)
$$

then $\left(a_{2}-b_{2}\right) f-\left(a_{1}-b_{1}\right) f^{\prime}+a_{1} b_{2}-a_{2} b_{1} \equiv 0$.

Proof. Assume that $\left(a_{2}-b_{2}\right) f-\left(a_{1}-b_{1}\right) f^{\prime}+a_{1} b_{2}-a_{2} b_{1} \not \equiv 0$. Let $\alpha, \beta, g, h$, and $\gamma_{i}$ be defined by (11)-(15), respectively. Then from the proof process of Lemma 3 we know $\alpha \neq \equiv 0, \beta \not \equiv 0$, $g \neq \equiv 0, h \neq \equiv 0, \gamma_{i} \neq \equiv a_{2}$, and $\gamma_{i} \neq \equiv b_{2}(i=1,2)$. Since $f$ and $f^{\prime}$ share $\left(a_{1}, a_{2}\right)$ and $\left(b_{1}, b_{2}\right)$ IM, by Lemmas 1,2 , and 3 it follows that

$$
\begin{gathered}
m(r, f)=\bar{N}\left(r, \frac{1}{f-a_{1}}\right)+\bar{N}\left(r, \frac{1}{f-b_{1}}\right)+S(r), \\
T(r, g)=S(r) \\
T(r, h) \leq T(r, f)-N\left(r, \frac{1}{f^{\prime}-\gamma_{i}}\right)+S(r), \quad i=1,2 .
\end{gathered}
$$

Now from the second fundamental theorem, (27), (28), and the assumption that $f$ is entire, we deduce

$$
\begin{aligned}
2 m\left(r, f^{\prime}\right)= & 2 T\left(r, f^{\prime}\right) \\
\leq & \bar{N}\left(r, \frac{1}{f^{\prime}-a_{2}}\right) \\
& +\bar{N}\left(r, \frac{1}{f^{\prime}-b_{2}}\right)+\bar{N}\left(r, \frac{1}{f^{\prime}-\gamma_{i}}\right)+S(r) \\
\leq & \bar{N}\left(r, \frac{1}{f-a_{1}}\right)+\bar{N}\left(r, \frac{1}{f-b_{1}}\right) \\
& +N\left(r, \frac{1}{f^{\prime}-\gamma_{i}}\right)+S(r) \\
= & m(r, f)+N\left(r, \frac{1}{f^{\prime}-\gamma_{i}}\right)+S(r) \\
= & m\left(r, f^{\prime}\right)+N\left(r, \frac{1}{f^{\prime}-\gamma_{i}}\right)+S(r),
\end{aligned}
$$

which yields

$$
N\left(r, \frac{1}{f^{\prime}-\gamma_{i}}\right)=m\left(r, f^{\prime}\right)+S(r) .
$$

Again by (27), (30), (32), and the assumption that $f$ is entire, we obtain

$$
T(r, h)=S(r) .
$$

For any $z_{0} \in S_{(m, n)}\left(a_{1}, a_{2}\right) \cup S_{(m, n)}\left(b_{1}, b_{2}\right)$, from (13) and (14), we can get $n g\left(z_{0}\right)-m h\left(z_{0}\right)=0$.
If $n g-m h \equiv 0$, then by (13) and (14) we deduce

$$
n\left(\frac{f^{\prime}-b_{1}^{\prime}}{f-b_{1}}-\frac{f^{\prime}-a_{1}^{\prime}}{f-a_{1}}\right) \equiv m\left(\frac{f^{\prime \prime}-b_{2}^{\prime}}{f^{\prime}-b_{2}}-\frac{f^{\prime \prime}-a_{2}^{\prime}}{f^{\prime}-a_{2}}\right),
$$

which implies that

$$
\left(\frac{f-b_{1}}{f-a_{1}}\right)^{n} \equiv c_{1}\left(\frac{f^{\prime}-b_{2}}{f^{\prime}-a_{2}}\right)^{m}
$$

where $c_{1}$ is a nonzero constant. If $n \neq m$, then from (35) and the condition that $f$ is entire, we obtain $n m(r, f)=$ $m m\left(r, f^{\prime}\right)+S(r)$, which contradicts (27). If $n=m$, then we get

$$
\frac{f-b_{1}}{f-a_{1}} \equiv c_{2}\left(\frac{f^{\prime}-b_{2}}{f^{\prime}-a_{2}}\right)
$$

where $c_{2}$ is a nonzero constant. We claim that $c_{2} \neq 1$. Indeed, if $c_{2}=1$, then by (36) we deduce

$$
\frac{f-b_{1}}{f-a_{1}} \equiv \frac{f^{\prime}-b_{2}}{f^{\prime}-a_{2}}
$$

which leads to $\left(a_{2}-b_{2}\right) f-\left(a_{1}-b_{1}\right) f^{\prime}+a_{1} b_{2}-a_{2} b_{1} \equiv 0$. This contradicts the assumption. Thus $c_{2} \neq 1$ and so from (36) we have

$$
f\left[\left(1-c_{2}\right) f^{\prime}+c_{2} b_{2}-a_{2}\right]=\left(b_{1}-c_{2} a_{1}\right) f^{\prime}+c_{2} a_{1} b_{2}-a_{2} b_{1} .
$$

This and Lemma 4 yield

$$
m\left(r,\left(1-c_{2}\right) f^{\prime}+c_{2} b_{2}-a_{2}\right)=S(r),
$$

which gives $m\left(r, f^{\prime}\right)=S(r)$. From this and the condition that $f$ is entire, it follows that $T\left(r, f^{\prime}\right)=S(r)$, a contradiction. Hence $n g-m h \neq 0$, for any positive integers $m$ and $n$.

Therefore by (29) and (33) we obtain

$$
\begin{aligned}
\bar{N}_{(m, n)} & \left(r, \frac{1}{f-a_{1}}\right)+\bar{N}_{(m, n)}\left(r, \frac{1}{f-b_{1}}\right) \\
\leq & N\left(r, \frac{1}{n g-m h}\right)+S(r) \\
\leq & T(r, g)+T(r, h)+S(r) \\
& =S(r),
\end{aligned}
$$


for any positive integers $m$ and $n$. It follows from this, Lemma 1 , the second fundamental theorem, and the condition that $f$ is entire that

$$
\begin{aligned}
& T(r, f) \\
& \leq \bar{N}\left(r, \frac{1}{f-a_{1}}\right)+\bar{N}\left(r, \frac{1}{f-b_{1}}\right)+S(r) \\
& =\sum_{m, n}\left(\bar{N}_{(m, n)}\left(r, \frac{1}{f-a_{1}}\right)+\bar{N}_{(m, n)}\left(r, \frac{1}{f-b_{1}}\right)\right)+S(r) \\
& =\sum_{m+n \geq 6}\left(\bar{N}_{(m, n)}\left(r, \frac{1}{f-a_{1}}\right)+\bar{N}_{(m, n)}\left(r, \frac{1}{f-b_{1}}\right)\right)+S(r) \\
& \leq \sum_{m+n \geq 6} \frac{1}{6}\left(N_{(m, n)}\left(r, \frac{1}{f-a_{1}}\right)\right. \\
& \left.+N_{(m, n)}\left(r, \frac{1}{f^{\prime}-a_{2}}\right)\right) \\
& +\sum_{m+n \geq 6} \frac{1}{6}\left(N_{(m, n)}\left(r, \frac{1}{f-b_{1}}\right)\right. \\
& \left.+N_{(m, n)}\left(r, \frac{1}{f^{\prime}-b_{2}}\right)\right)+S(r) \\
& \leq \frac{1}{6}\left(N\left(r, \frac{1}{f-a_{1}}\right)+N\left(r, \frac{1}{f^{\prime}-a_{2}}\right)\right) \\
& +\frac{1}{6}\left(N\left(r, \frac{1}{f-b_{1}}\right)+N\left(r, \frac{1}{f^{\prime}-b_{2}}\right)\right)+S(r) \\
& \leq \frac{1}{3} T(r, f)+\frac{1}{3} T\left(r, f^{\prime}\right)+S(r) \\
& =\frac{1}{3} T(r, f)+\frac{1}{3} m\left(r, f^{\prime}\right)+S(r) \\
& =\frac{1}{3} T(r, f)+\frac{1}{3} m\left(r, \frac{f^{\prime}}{f} f\right)+S(r) \\
& \leq \frac{1}{3} T(r, f)+\frac{1}{3} m(r, f)+S(r) \\
& =\frac{1}{3} T(r, f)+\frac{1}{3} T(r, f)+S(r) \\
& =\frac{2}{3} T(r, f)+S(r) \text {, }
\end{aligned}
$$

which implies that $T(r, f)=S(r)$, a contradiction. Thus $\left(a_{2}-\right.$ $\left.b_{2}\right) f-\left(a_{1}-b_{1}\right) f^{\prime}+a_{1} b_{2}-a_{2} b_{1} \equiv 0$.

This completes the proof of Lemma 5.

\section{Proof of Theorem 1}

Suppose that $\left(a_{2}-b_{2}\right) f-\left(a_{1}-b_{1}\right) f^{\prime}+a_{1} b_{2}-a_{2} b_{1} \not \equiv 0$. Since $f$ and $f^{\prime}$ share $\left(a_{1}, a_{2}\right)$ and $\left(b_{1}, b_{2}\right)$ IM, by Lemma 1 we have $S(r, f)=S\left(r, f^{\prime}\right):=S(r)$. Let $\alpha, \beta, g, h$, and $\gamma_{i}$ be defined by (11)-(15), respectively. Then from the proof process of Lemma 3 we know $\alpha \neq \equiv 0, \beta \not \equiv 0, g \neq \equiv 0, h \not \equiv 0, \gamma_{i} \neq \equiv a_{2}$, and $\gamma_{i} \neq b_{2}(i=1,2)$. Next we rewrite (13) as

$$
\begin{aligned}
& {\left[g-\left(a_{1}^{\prime}-b_{1}^{\prime}\right)\left(a_{2}-b_{2}\right)\right] f^{2}} \\
& \quad=d_{1} f f^{\prime}+d_{2} f+d_{3} f^{\prime}+d_{4} f^{\prime 2}+d_{5}
\end{aligned}
$$

where $d_{1}=\left(a_{1}-b_{1}\right)\left(b_{1}^{\prime}+b_{2}-a_{2}-a_{1}^{\prime}\right), d_{2}=\left(a_{1}^{\prime}-b_{1}^{\prime}\right)\left(a_{1} b_{2}-\right.$ $\left.a_{2} b_{1}\right)+\left(a_{2}-b_{2}\right)\left(a_{1} b_{1}^{\prime}-a_{1}^{\prime} b_{1}\right)+\left(a_{1}+b_{1}\right) g, d_{3}=\left(a_{1}-b_{1}\right)\left(a_{2} b_{1}+\right.$ $\left.a_{1}^{\prime} b_{1}-a_{1} b_{2}-a_{1} b_{1}^{\prime}\right), d_{4}=\left(a_{1}-b_{1}\right)^{2}$, and $d_{5}=\left(a_{1} b_{1}^{\prime}-a_{1}^{\prime} b_{1}\right)\left(a_{1} b_{2}-\right.$ $\left.a_{2} b_{1}\right)-a_{1} b_{1} g$ are all small functions with respect to $f$.

Now we divide into two cases.

Case 1. $g-\left(a_{1}^{\prime}-b_{1}^{\prime}\right)\left(a_{2}-b_{2}\right) \equiv 0$; that is, $g \equiv\left(a_{1}^{\prime}-b_{1}^{\prime}\right)\left(a_{2}-b_{2}\right)$. We again discuss the three subcases.

Subcase 1. $a_{1}^{\prime} \not \equiv a_{2}$ and $b_{1}^{\prime} \not \equiv b_{2}$. Since $f$ and $f^{\prime}$ share $\left(a_{1}, a_{2}\right)$ and $\left(b_{1}, b_{2}\right)$ IM, the zeros of $f-a_{1}$ and $f-b_{1}$ of multiplicity larger than one are the zeros $a_{1}^{\prime}-a_{2}$ and $b_{1}^{\prime}-b_{2}$, respectively. It then follows that

$$
\begin{aligned}
& \sum_{m \geq 2, n \geq 1}\left(\bar{N}_{(m, n)}\left(r, \frac{1}{f-a_{1}}\right)+\bar{N}_{(m, n)}\left(r, \frac{1}{f-b_{1}}\right)\right) \\
& \leq N\left(r, \frac{1}{a_{1}^{\prime}-a_{2}}\right)+N\left(r, \frac{1}{b_{1}^{\prime}-b_{2}}\right)+S(r) \\
& \leq T\left(r, a_{1}^{\prime}\right)+T\left(r, a_{2}\right)+T\left(r, b_{1}^{\prime}\right) \\
&+T\left(r, b_{2}\right)+S(r) \\
&= S(r) ;
\end{aligned}
$$

that is,

$$
\sum_{m \geq 2, n \geq 1}\left(\bar{N}_{(m, n)}\left(r, \frac{1}{f-a_{1}}\right)+\bar{N}_{(m, n)}\left(r, \frac{1}{f-b_{1}}\right)\right)=S(r) .
$$

Let $z_{0} \in S_{(1, n)}\left(a_{1}, a_{2}\right)$. For $n \geq 2$, from (13) we get

$$
\begin{aligned}
g\left(z_{0}\right) & =\left(a_{1}^{\prime}\left(z_{0}\right)-a_{2}\left(z_{0}\right)\right)\left(a_{2}\left(z_{0}\right)-b_{2}\left(z_{0}\right)\right) \\
& =\left(a_{1}^{\prime}\left(z_{0}\right)-b_{1}^{\prime}\left(z_{0}\right)\right)\left(a_{2}\left(z_{0}\right)-b_{2}\left(z_{0}\right)\right)
\end{aligned}
$$

which implies that $a_{2}\left(z_{0}\right)-b_{1}^{\prime}\left(z_{0}\right)=0$ or $a_{2}\left(z_{0}\right)-b_{2}\left(z_{0}\right)=0$.

If $a_{2}-b_{1}^{\prime} \equiv 0$, then by (13) we deduce

$$
f^{\prime}=\frac{a_{1}^{\prime}-b_{2}}{a_{1}-b_{1}} f+\frac{a_{1} b_{2}-a_{1}^{\prime} b_{1}}{a_{1}-b_{1}}
$$

which, in view of the condition that $f$ is entire, implies that $m(r, f)=m\left(r, f^{\prime}\right)+S(r)$. From this and Lemma 5, it follows that $\left(a_{2}-b_{2}\right) f-\left(a_{1}-b_{1}\right) f^{\prime}+a_{1} b_{2}-a_{2} b_{1} \equiv 0$, contradicting the assumption. Thus $a_{2}-b_{1}^{\prime} \not \equiv 0$. By the conditions in Theorem 1 , we know that $a_{2}-b_{2} \not \equiv 0$. 
Hence

$$
\begin{aligned}
& \sum_{n \geq 2} \bar{N}_{(1, n)}\left(r, \frac{1}{f-a_{1}}\right) \\
& \quad \leq N\left(r, \frac{1}{a_{2}-b_{1}^{\prime}}\right)+N\left(r, \frac{1}{a_{2}-b_{2}}\right)+S(r) \\
& \quad \leq 2 T\left(r, a_{2}\right)+T\left(r, b_{2}\right)+T\left(r, b_{1}^{\prime}\right)+S(r)=S(r) ;
\end{aligned}
$$

that is,

$$
\sum_{n \geq 2} \bar{N}_{(1, n)}\left(r, \frac{1}{f-a_{1}}\right)=S(r)
$$

Similarly,

$$
\sum_{n \geq 2} \bar{N}_{(1, n)}\left(r, \frac{1}{f-b_{1}}\right)=S(r) .
$$

It then follows from (44)-(49) and the second fundamental theorem that

$$
\begin{aligned}
T(r, f) & \leq \bar{N}\left(r, \frac{1}{f-a_{1}}\right)+\bar{N}\left(r, \frac{1}{f-b_{1}}\right)+S(r) \\
& =\bar{N}_{(1,1)}\left(r, \frac{1}{f-a_{1}}\right)+\bar{N}_{(1,1)}\left(r, \frac{1}{f-b_{1}}\right)+S(r) .
\end{aligned}
$$

For any $z_{1} \in S_{(1,1)}\left(a_{1}, a_{2}\right) \cup S_{(1,1)}\left(b_{1}, b_{2}\right)$, from (13) and (14), we can get $g\left(z_{1}\right)-h\left(z_{1}\right)=0$.

If $g-h \equiv 0$, then by (13) and (14) we have

$$
\frac{f^{\prime}-b_{1}^{\prime}}{f-b_{1}}-\frac{f^{\prime}-a_{1}^{\prime}}{f-a_{1}} \equiv \frac{f^{\prime \prime}-b_{2}^{\prime}}{f^{\prime}-b_{2}}-\frac{f^{\prime \prime}-a_{2}^{\prime}}{f^{\prime}-a_{2}},
$$

which implies that

$$
\frac{f-b_{1}}{f-a_{1}} \equiv c_{3} \frac{f^{\prime}-b_{2}}{f^{\prime}-a_{2}},
$$

where $c_{3}$ is a nonzero constant. We claim that $c_{3} \neq 1$. Indeed, if $c_{3}=1$, then by (52) we have

$$
\frac{f-b_{1}}{f-a_{1}} \equiv \frac{f^{\prime}-b_{2}}{f^{\prime}-a_{2}},
$$

which leads to $\left(a_{2}-b_{2}\right) f-\left(a_{1}-b_{1}\right) f^{\prime}+a_{1} b_{2}-a_{2} b_{1} \equiv 0$. This contradicts the assumption. Thus $c_{3} \neq 1$ and so from (52) we get

$$
f\left[\left(1-c_{3}\right) f^{\prime}+c_{3} b_{2}-a_{2}\right]=\left(b_{1}-c_{3} a_{1}\right) f^{\prime}+c_{3} a_{1} b_{2}-a_{2} b_{1} .
$$

This and Lemma 4 yield

$$
m\left(r,\left(1-c_{3}\right) f^{\prime}+c_{3} b_{2}-a_{2}\right)=S(r),
$$

which gives $m\left(r, f^{\prime}\right)=S(r)$. From this and the condition that $f$ is entire, it follows that $T\left(r, f^{\prime}\right)=S(r)$, a contradiction. Hence $g-h \neq 0$.

Therefore by (50) and Lemma 3 we obtain

$$
\begin{aligned}
T(r, f) & \leq \bar{N}_{(1,1)}\left(r, \frac{1}{f-a_{1}}\right)+\bar{N}_{(1,1)}\left(r, \frac{1}{f-b_{1}}\right)+S(r) \\
& \leq N\left(r, \frac{1}{g-h}\right)+S(r) \\
& \leq T(r, g)+T(r, h)+S(r) \\
& \leq T(r, f)-N\left(r, \frac{1}{f^{\prime}-\gamma_{i}}\right)+S(r)
\end{aligned}
$$

which implies that

$$
N\left(r, \frac{1}{f^{\prime}-\gamma_{i}}\right)=S(r), \quad i=1,2 .
$$

This is impossible by the second fundamental theorem.

Subcase 2. Either $a_{1}^{\prime} \equiv a_{2}$ and $b_{1}^{\prime} \not \equiv b_{2}$ or $a_{1}^{\prime} \neq \equiv a_{2}$ and $b_{1}^{\prime} \equiv b_{2}$. Without loss of generality, we assume that $a_{1}^{\prime} \equiv a_{2}$ and $b_{1}^{\prime} \not \equiv$ $b_{2}$. It is easy to see by (13) that the zeros of $f-a_{1}$ and $f^{\prime}-a_{2}$ of multiplicity all larger than one are the zeros of $g$. Thus by Lemma 3,

$$
\begin{aligned}
\sum_{m \geq 2, n \geq 2} \bar{N}_{(m, n)}\left(r, \frac{1}{f-a_{1}}\right) & \leq N\left(r, \frac{1}{g}\right)+S(r) \\
& \leq T(r, g)+S(r)=S(r)
\end{aligned}
$$

that is,

$$
\sum_{m \geq 2, n \geq 2} \bar{N}_{(m, n)}\left(r, \frac{1}{f-a_{1}}\right)=S(r) .
$$

By the discussion of Subcase 1, we see

$$
\begin{aligned}
\bar{N}\left(r, \frac{1}{f-b_{1}}\right) & =\bar{N}_{(1,1)}\left(r, \frac{1}{f-b_{1}}\right)+S(r) \\
& \leq N\left(r, \frac{1}{g-h}\right)+S(r) \\
& \leq T(r, g)+T(r, h)+S(r) \\
& \leq T(r, f)-N\left(r, \frac{1}{f^{\prime}-\gamma_{i}}\right)+S(r) ;
\end{aligned}
$$

that is,

$$
\begin{array}{r}
\bar{N}\left(r, \frac{1}{f-b_{1}}\right) \leq T(r, f)-N\left(r, \frac{1}{f^{\prime}-\gamma_{i}}\right) \\
+S(r), \\
i=1,2 .
\end{array}
$$

Note that the zeros of $f-a_{1}$ of multiplicity larger than one are all the zeros of $f^{\prime}-a_{2}=f^{\prime}-a_{1}^{\prime}$. Since $f$ and $f^{\prime}$ share $\left(a_{1}, a_{2}\right) \mathrm{IM}$, it follows that

$$
\bar{N}_{(1,1)}\left(r, \frac{1}{f-a_{1}}\right)=S(r) .
$$


Now from (59)-(62) and the second fundamental theorem, we obtain

$$
\begin{aligned}
T(r, f) \leq & \bar{N}\left(r, \frac{1}{f-a_{1}}\right)+\bar{N}\left(r, \frac{1}{f-b_{1}}\right)+S(r) \\
\leq & \bar{N}_{(2,1)}\left(r, \frac{1}{f-a_{1}}\right)+T(r, f) \\
& -N\left(r, \frac{1}{f^{\prime}-\gamma_{i}}\right)+S(r)
\end{aligned}
$$

that is,

$$
N\left(r, \frac{1}{f^{\prime}-\gamma_{i}}\right) \leq \bar{N}_{(2,1)}\left(r, \frac{1}{f-a_{1}}\right)+S(r), \quad i=1,2 .
$$

Let

$$
\phi=2 \frac{f^{\prime \prime}-b_{2}^{\prime}}{f^{\prime}-b_{2}}-2 \frac{f^{\prime}-b_{1}^{\prime}}{f-b_{1}}+\frac{a_{1}^{\prime}-b_{1}^{\prime}}{a_{1}-b_{1}}-2 \frac{a_{2}^{\prime}-b_{2}^{\prime}}{a_{2}-b_{2}} .
$$

It is easily seen from (65) and the lemma of the logarithmic derivative that

$$
m(r, \phi)=S(r)
$$

Note that common simple zeros of $f-b_{1}$ and $f^{\prime}-b_{2}$ are not the poles of $\phi$. In terms of the discussion of Subcase 1, from (65) we know $N(r, \phi)=S(r)$, which together with (66) gives that

$$
T(r, \phi)=S(r)
$$

Let $z_{2} \in S_{(2,1)}\left(a_{1}, a_{2}\right)$. Then by (65) and (13) we have $\phi\left(z_{2}\right)=0$.

If $\phi \equiv 0$, then from (65) we derive

$$
\left(f^{\prime}-b_{2}\right)^{2}=c_{4} \frac{\left(a_{2}-b_{2}\right)^{2}}{a_{1}-b_{1}}\left(f-b_{1}\right)^{2}
$$

where $c_{4}$ is a nonzero constant. This, in view of the condition that $f$ is entire, implies that $m(r, f)=m\left(r, f^{\prime}\right)+S(r)$. From this and Lemma 5 , it follows that $\left(a_{2}-b_{2}\right) f-\left(a_{1}-b_{1}\right) f^{\prime}+$ $a_{1} b_{2}-a_{2} b_{1} \equiv 0$, contradicting the assumption. Thus $\phi \not \equiv 0$.

Hence by (64) and (67) we obtain

$$
\begin{aligned}
N\left(r, \frac{1}{f^{\prime}-\gamma_{i}}\right) & \leq \bar{N}_{(2,1)}\left(r, \frac{1}{f-a_{1}}\right)+S(r) \\
& \leq N\left(r, \frac{1}{\phi}\right)+S(r) \\
& \leq T(r, \phi)+S(r) \\
& \leq S(r) ;
\end{aligned}
$$

that is,

$$
N\left(r, \frac{1}{f^{\prime}-\gamma_{i}}\right)=S(r), \quad i=1,2 .
$$

This is also impossible by the second fundamental theorem.
Subcase 3. $a_{1}^{\prime} \equiv a_{2}$ and $b_{1}^{\prime} \equiv b_{2}$. By the discussion of Subcase 2, we see

$$
T(r, f) \leq \bar{N}_{(2,1)}\left(r, \frac{1}{f-a_{1}}\right)+\bar{N}_{(2,1)}\left(r, \frac{1}{f-b_{1}}\right)+S(r) .
$$

We claim that

$$
\begin{aligned}
& \bar{N}_{(2,1)}\left(r, \frac{1}{f-a_{1}}\right)=S(r), \\
& \bar{N}_{(2,1)}\left(r, \frac{1}{f-b_{1}}\right)=S(r) .
\end{aligned}
$$

Let

$$
\psi=2 \frac{f^{\prime \prime}-b_{2}^{\prime}}{f^{\prime}-b_{2}}-\frac{f^{\prime}-b_{1}^{\prime}}{f-b_{1}}-2 \frac{a_{2}^{\prime}-b_{2}^{\prime}}{a_{2}-b_{2}} .
$$

It is easily known from (74) and the lemma of the logarithmic derivative that

$$
m(r, \psi)=S(r) .
$$

Note that common zeros of $f-b_{1}$ of multiplicity two and $f^{\prime}-b_{2}$ of multiplicity one are not the poles of $\psi$. In terms of the discussion of Subcase 2, we know $N(r, \psi)=S(r)$, which together with (75) gives that

$$
T(r, \psi)=S(r) .
$$

Let $z_{3} \in S_{(2,1)}\left(a_{1}, a_{2}\right)$. Then by (74) and (13) we have $\psi\left(z_{3}\right)=0$.

If $\psi \neq \equiv 0$, then from (76) we get

$$
\begin{aligned}
\bar{N}_{(2,1)}\left(r, \frac{1}{f-a_{1}}\right) & \leq N\left(r, \frac{1}{\psi}\right)+S(r) \\
& \leq T(r, \psi)+S(r) \leq S(r),
\end{aligned}
$$

that is,

$$
\bar{N}_{(2,1)}\left(r, \frac{1}{f-a_{1}}\right)=S(r) .
$$

If $\psi \equiv 0$, then by (74) we deduce

$$
\left(f^{\prime}-b_{2}\right)^{2}=c_{5}\left(a_{2}-b_{2}\right)^{2}\left(f-b_{1}\right)
$$

where $c_{5}$ is a nonzero constant. This implies $a_{1}\left(z_{3}\right)-b_{1}\left(z_{3}\right)-$ $1 / c_{5}=0$. Since $a_{1}^{\prime} \equiv a_{2}, b_{1}^{\prime} \equiv b_{2}$, and $a_{2} \not \equiv b_{2}$, we obtain $a_{1}-b_{1}-1 / c_{5} \neq \equiv 0$. Thus

$$
\begin{aligned}
\bar{N}_{(2,1)}\left(r, \frac{1}{f-a_{1}}\right) & \leq N\left(r, \frac{1}{a_{1}-b_{1}-1 / c_{5}}\right)+S(r) \\
& \leq T\left(r, a_{1}\right)+T\left(r, b_{1}\right)+S(r) \leq S(r) ;
\end{aligned}
$$

that is,

$$
\bar{N}_{(2,1)}\left(r, \frac{1}{f-a_{1}}\right)=S(r) .
$$

Hence (72) follows. In the same manner as above, we can prove (73). The proof of the claim is complete. Now by (71)(73) we get $T(r, f)=S(r)$, a contradiction. 
Case 2. $g-\left(a_{1}^{\prime}-b_{1}^{\prime}\right)\left(a_{2}-b_{2}\right) \not \equiv 0$. Then by (42) and (i) in Lemma 3 we have

$$
\begin{aligned}
2 m(r, f) \leq & m\left(r, \frac{1}{g-\left(a_{1}^{\prime}-b_{1}^{\prime}\right)\left(a_{2}-b_{2}\right)}\right) \\
& +m\left(r, d_{1} f f^{\prime}+d_{2} f+d_{3} f^{\prime}+d_{4} f^{\prime 2}+d_{5}\right) \\
\leq & m\left(r, f\left(d_{1} f^{\prime}+d_{2}+d_{3} \frac{f^{\prime}}{f}+d_{4} f^{\prime} \frac{f^{\prime}}{f}\right)\right) \\
& +m\left(r, d_{5}\right)+S(r) \\
\leq & m(r, f)+m\left(r, f^{\prime}\left(d_{1}+d_{4} \frac{f^{\prime}}{f}\right)\right)+S(r) \\
\leq & m(r, f)+m\left(r, f^{\prime}\right)+S(r),
\end{aligned}
$$

which implies that

$$
m(r, f) \leq m\left(r, f^{\prime}\right)+S(r)
$$

On the other hand,

$$
m\left(r, f^{\prime}\right) \leq m\left(r, f \frac{f^{\prime}}{f}\right) \leq m(r, f)+S(r) .
$$

Combining (83) with (84) yields

$$
m(r, f)=m\left(r, f^{\prime}\right)+S(r) .
$$

This and Lemma 5 lead to $\left(a_{2}-b_{2}\right) f-\left(a_{1}-b_{1}\right) f^{\prime}+a_{1} b_{2}-a_{2} b_{1} \equiv$ 0 , contradicting the assumption.

This completes the proof of Theorem 1.

\section{Conflict of Interests}

The author declares that there is no conflict of interests regarding the publication of this paper.

\section{Acknowledgments}

The author would like to thank the referees for their thorough comments and helpful suggestions. Project supported by the National Natural Science Foundation of China (Grant no. 11301076), the Natural Science Foundation of Fujian Province, China (Grant no. 2014J01004), the Education Department Foundation of Fujian Province, China (Grant no. JB13018), and the Innovation Team of Nonlinear Analysis and its Applications of Fujian Normal University, China (Grant no. IRTL1206).

\section{References}

[1] W. K. Hayman, Meromorphic Functions, Clarendon Press, Oxford, UK, 1964.

[2] L. Yang, Value Distribution Theory, Springer, Berlin, Germany, 1993.
[3] C. C. Yang and H. X. Yi, Uniqueness Theory of Meromorphic Functions, vol. 557, Kluwer Academic Publishers, Dordrecht, The Netherlands, 2003.

[4] I. Laine, Nevanlinna Theory and Complex Differential Equations, Walter De Gruyter, Berlin, Germany, 1993.

[5] L. A. Rubel and C. C. Yang, "Values shared by an entire function and its derivative," in Complex Analysis, vol. 599 of Lecture Notes in Mathematics, pp. 101-103, Springer, Berlin, Germany, 1977.

[6] E. Mues and N. Steinmetz, "Meromorphe Funktionen, die mit ihrer Ableitung Werte teilen," Manuscripta Mathematica, vol. 29, no. 2-4, pp. 195-206, 1979.

[7] J. H. Zheng and S. P. Wang, "On the unicity of meromorphic functions and their derivatives," Advances in Mathematics, vol. 21, no. 3, pp. 334-341, 1992.

[8] G. Qiu, "Uniqueness of entire functions that share some small functions," Kodai Mathematical Journal, vol. 23, no. 1, pp. 1-11, 2000.

[9] P. Li, "Unicity of meromorphic functions and their derivatives," Journal of Mathematical Analysis and Applications, vol. 285, no. 2, pp. 651-665, 2003.

[10] J. Clunie, “On integral and meromorphic functions," Journal of the London Mathematical Society, vol. 37, pp. 17-27, 1962.

[11] E. Mues and N. Steinmetz, "The theorem of Tumura-Clunie for meromorphic functions," Journal of the London Mathematical Society, vol. 23, no. 1, pp. 113-122, 1981.

[12] G. Weissenborn, "On the theorem of Tumura and Clunie," The Bulletin of the London Mathematical Society, vol. 18, no. 4, pp. 371-373, 1986. 


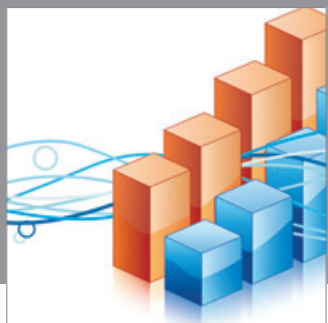

Advances in

Operations Research

mansans

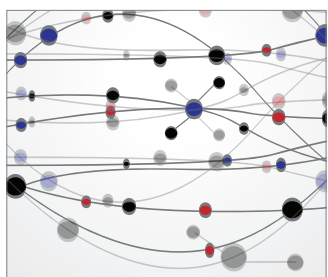

The Scientific World Journal
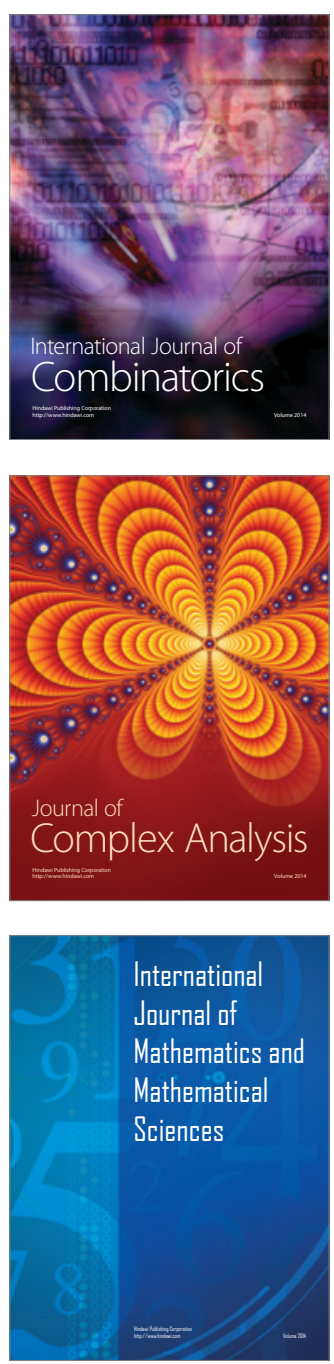
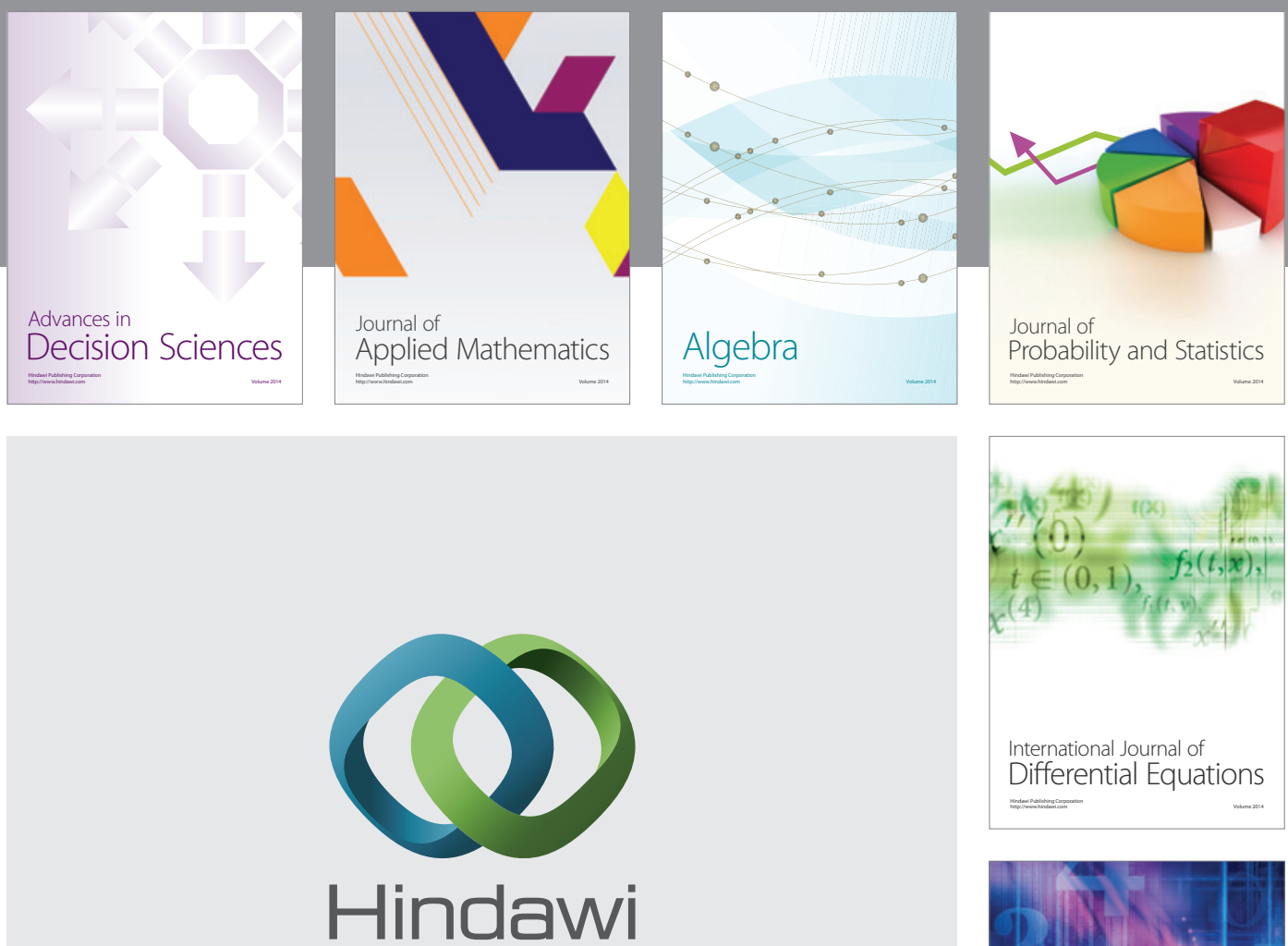

Submit your manuscripts at http://www.hindawi.com
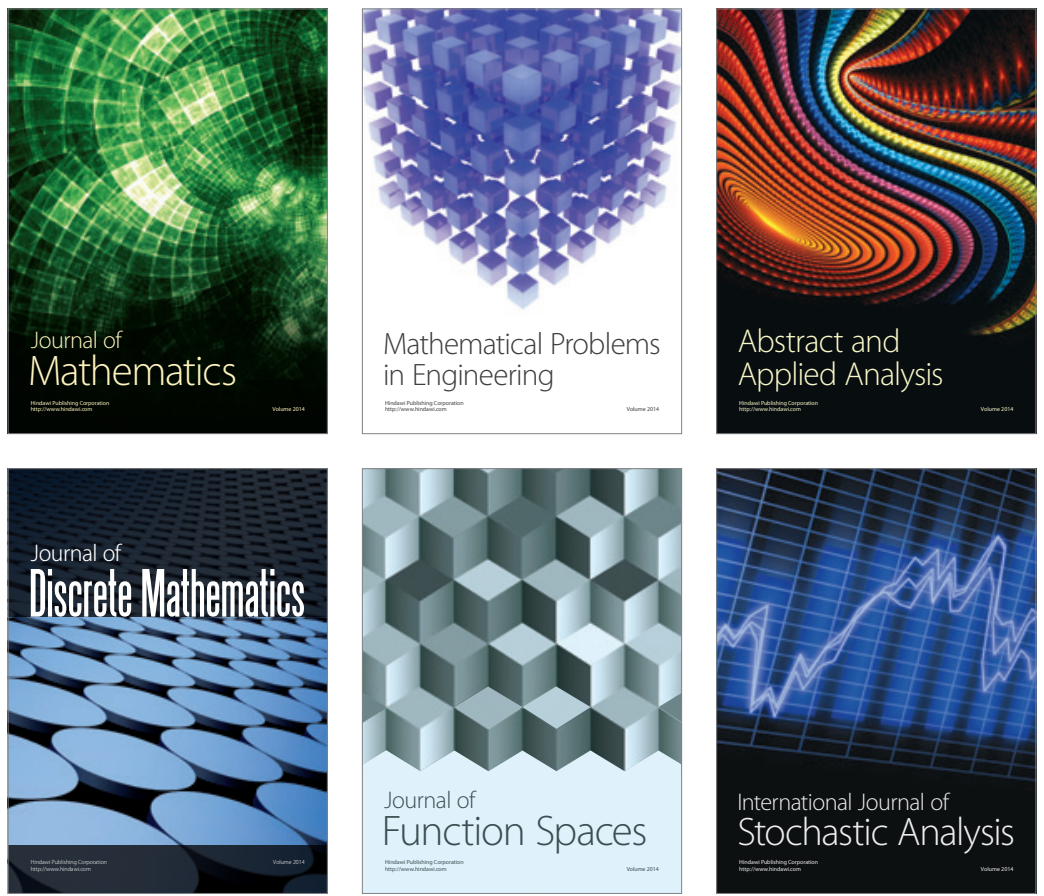

Journal of

Function Spaces

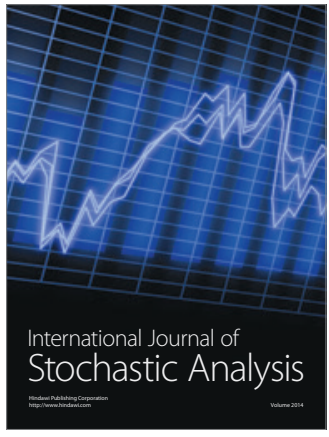

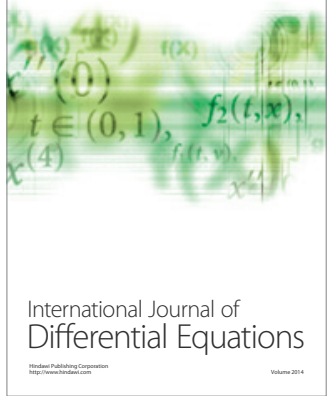
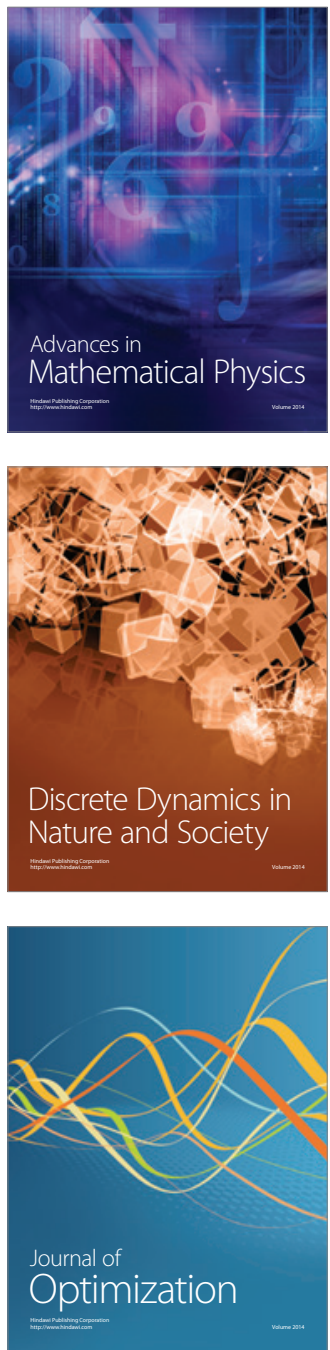